\title{
EDITORIAL
}

\section{The potential of brain stimulation techniques for substance use disorder treatment}

\author{
Heather E. Webber, (iD Joy M. Schmitz iD \\ Faillace Department of Psychiatry and Behavioral Sciences, University of Texas Health Science Center at Houston, TX, USA.
}

Current treatments for substance use disorders (SUDs) are far from ideal, with no U.S. Food and Drug Administration-approved pharmacotherapies to treat stimulant and other use disorders. New approaches are urgently needed to combat the increasing prevalence of SUDs and overdose deaths. It is widely accepted that addiction is a chronic disease of the brain involving changes in neural circuitry associated with cognitive and reward functioning. Brain stimulation techniques are novel methods that can modulate these circuits directly and thus hold considerable promise for treating SUDs. Below we briefly describe three of these neuromodulation techniques (see Box 1 for summary).

Transcranial direct current stimulation (tDCS) is a noninvasive technique that uses an anode and cathode to apply a low intensity (1-2 mA) constant current to a superficial area of the brain. Anodal tDCS increases and cathodal tDCS decreases cortical excitability, although there are individual differences. Despite the fact that the exact mechanisms of tDCS are unknown, its advantages include low cost, minimal risk, and easy application. The most common area targeted with tDCS is the dorsolateral prefrontal cortex (dIPFC), given its role in decisionmaking, working memory, and emotion and its position directly under the scalp. In a systematic review of 16 clinical trials investigating tDCS of the dIPFC, ${ }^{4}$ eight studies provided evidence of reduced drug craving after stimulation. A handful of studies also reported reductions in cue reactivity, risky decision-making, and substance use. The studies varied on anodal and cathodal electrode placement, leaving unanswered questions about optimal stimulation. Future studies that use multi-session, shamcontrolled designs combining tDCS with neuroimaging and long-term follow-up assessment will be beneficial to understanding the mechanisms of tDCS.

Transcranial magnetic stimulation (TMS) is a noninvasive brain stimulation technique that uses an insulated coil to generate magnetic fields that pass through the scalp and skull unimpeded. These magnetic fields generate an electrical current that briefly modulates the neurons directly under the coil. When the pulses are repeated several times within a period of time (repetitive

Correspondence: Heather E. Webber, Faillace Department of Psychiatry and Behavioral Sciences, University of Texas Health Science Center at Houston, 1941 East Rd Houston, TX 77054, USA. E-mail: Heather.E.Webber@uth.tmc.edu

Submitted Nov 08 2021, accepted Nov 10 2021, Epub Jan 192022.
TMS [rTMS]), there can be a longer lasting response. rTMS likely works through long term potentiation/depression, with higher frequency stimulation generally having excitatory effects and low frequencies having inhibitory effects. TMS is well-tolerated and has a favorable safety profile. Considering the overlap in neural circuit disruptions, the majority of research has mimicked the depression literature, targeting the dIPFC as a treatment for SUD. In a review on sham-controlled rTMS studies for SUDs, ${ }^{5}$ excitatory rTMS to the dIPFC reduced craving and drug use in patients with cocaine and tobacco use disorders, but was less efficacious for alcohol. Notably, stimulation of the dIPFC did not consistently show reductions in both substance use and craving, suggesting the need to consider alternative target areas. Theoretically, increasing activity in areas involved in cognitive control and response inhibition, such as the dorsomedial PFC and the dorsal anterior cingulate cortex, and decreasing activity in areas associated with drug reward reactivity, such as the ventromedial PFC (vmPFC), could be beneficial for treating addiction. ${ }^{6}$ Secondary measures, such as cognitive functioning and neuroimaging, will be critical to our understanding of rTMS for SUD. While it is difficult to reach deeper areas, new coils are being developed. In fact, BrainsWay's "deep TMS" H4coil, which targets the bilateral insula and PFC, received approval for smoking cessation in 2020.

Deep brain stimulation (DBS) involves implanting electrodes in the brain that send electrical pulses that modulate abnormal brain activity. Stimulation is controlled by a pacemaker-like device under the skin. DBS carries the risks associated with any neurosurgical procedure, but has important advantages over other non-invasive stimulation techniques, including the ability to easily alter stimulation parameters, stimulate deeper areas, and for the patient, reduce the burden associated with long-term care visits. The primary target for DBS treatment is the nucleus accumbens, given its involvement in drugseeking behavior, craving, and withdrawal. A recent systematic review of 11 studies (33 patients) targeting the nucleus accumbens reported average remission rates of $61 \%$ at 6 months and $53 \%$ at 1 year, ${ }^{7}$ along with

How to cite this article: Webber HE, Schmitz JM. The potential of brain stimulation techniques for substance use disorder treatment. Braz J Psychiatry. 2022;44:239-241. http://dx.doi.org/10.1590/15164446-2021-0040 


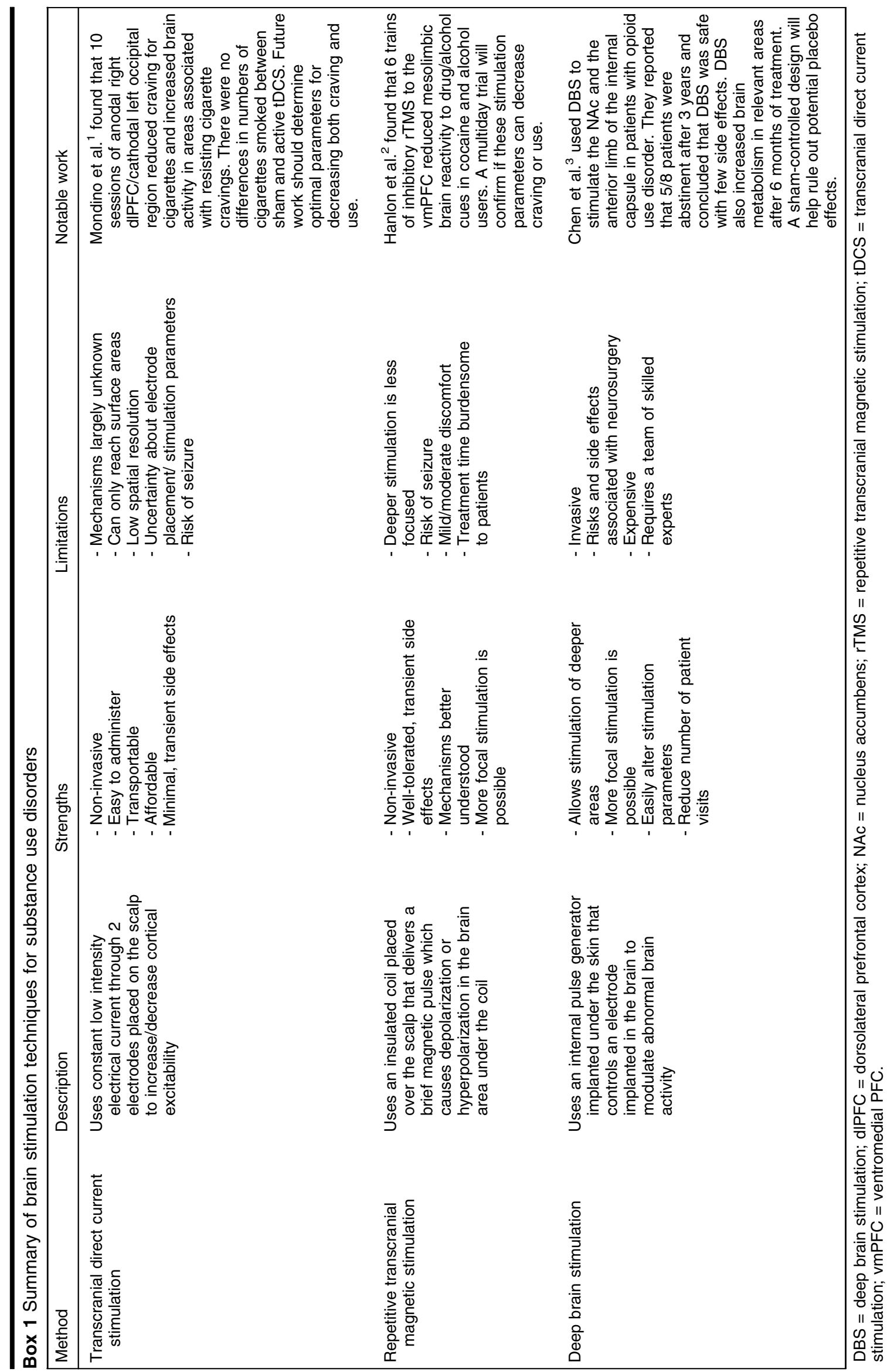


improvements in quality of life and drug craving. Although all of the studies in this review were case reports or series, ClinicalTrials.gov currently lists 15 active DBS trials at several international locations.

Despite its promise, the emerging field of neuromodulation treatments for SUD faces challenges. Defining the best "dose" is a critical question and a barrier to success. rTMS, for example, lacks clarity about ideal stimulation parameters, such as the number of pulses per session or sessions per day. Further, every substance has slightly different effects on neurotransmitter systems, suggesting that a more nuanced strategy may be needed for specific types of SUDs. Future research must also reconcile the effects of individual differences and state-dependent factors on treatment response. Brain stimulation could have increased efficacy when the brain is already in a particular state, ${ }^{8}$ and cognitive tasks/psychotherapy/ pharmacotherapy could be employed to induce brain states during brain stimulation to improve treatment outcomes. One of the most critical challenges involves the ability to safely engage neural targets in deep brain regions. DBS has a higher risk/benefit ratio, and TMS is limited by the principle that the deeper the magnetic field penetrates, the less focused the stimulation will be. Transcranial focused ultrasound is a cutting-edge technique that passes sound waves through the skull to modulate any brain area with exceptional precision. While there is no clinical data on focused ultrasound and SUD, this is an extremely exciting field of research that might address some of the challenges we face with current brain stimulation techniques.

\section{Disclosure}

The authors report no conflicts of interest.

\section{References}

1 Mondino M, Luck D, Grot S, Januel D, Suaud-Chagny MF, Poulet E, et al. Effects of repeated transcranial direct current stimulation on smoking, craving and brain reactivity to smoking cues. Sci Rep. 2018;8:8724.

2 Hanlon CA, Dowdle LT, Correia B, Mithoefer O, Kearney-Ramos T, Lench $D$, et al. Left frontal pole theta burst stimulation decreases orbitofrontal and insula activity in cocaine users and alcohol users. Drug Alcohol Depend. 2017;178:310-7.

3 Chen L, Li N, Ge S, Lozano AM, Lee DJ, Yang C, et al. Long-term results after deep brain stimulation of nucleus accumbens and the anterior limb of the internal capsule for preventing heroin relapse: an open-label pilot study. Brain Stimul. 2019;12:175-83.

4 Lupi M, Martinotti G, Santacroce R, Cinosi E, Carlucci M, Marini S, et al. Transcranial direct current stimulation in substance use disorders: a systematic review of scientific literature. J ECT. 2017;33:203-9.

5 Antonelli M, Fattore L, Sestito L, Di Giuda D, Diana M, Addolorato G. Transcranial Magnetic Stimulation: a review about its efficacy in the treatment of alcohol, tobacco and cocaine addiction. Addict Behav. 2021;114:106760.

6 Hanlon CA, Dowdle LT, Henderson JS. Modulating neural circuits with transcranial magnetic stimulation: implications for addiction treatment development. Pharmacol Rev. 2018;70:661-83.

7 Hassan O, Phan S, Wiecks N, Joaquin C, Bondarenko V. Outcomes of deep brain stimulation surgery for substance use disorder: a systematic review. Neurosurg Rev. 2021;44:1967-76.

8 Spagnolo PA, Montemitro C, Pettorruso M, Martinotti G, Di Giannantonio M. Better together? Coupling pharmacotherapies and cognitive interventions with non-invasive brain stimulation for the treatment of addictive disorders. Front Neurosci. 2020;13:1385. 